\title{
Effect of Polyols on the Interaction of $p$-Hydroxybenzoic Acid Esters with Polyoxyethylene Dodecyl Ether
}

\author{
Tsugio Shimamoto and Hiroyuki Mrma \\ Central Research Division, Takeda Chemical Industries, Ltd. ${ }^{1}$ )
}

(Received February 21, 1979)

\begin{abstract}
The effect of polyols, i.e., glycerol, propylene glycol and 1,3-butylene glycol, on the interaction between $p$-hydroxybenzoates and polyoxyethylene (15 units of oxyethylene) dodecyl ether was investigated by an ultrafiltration technique using a Diaflo membrane. Polyols had little effect on the binding of $p$-hydroxybenzoate to the primary class of sites in the nonionic surfactant micelles. In this binding process, bound preservative molecules are thought to be located at the oxyethylene-hydrocarbon interface, so it seems possible that polyols added to the system were too hydrophilic to penetrate deeply into the micelles and to compete effectively with this class of bound preservative. However, polyols modified the binding to the secondary class of sites to some extent; namely, these compounds could displace a part of the bound preservative from the polyoxyethylene region of the micelles. 1,3-Butylene glycol was the most effective polyol; glycerol was somewhat less effective in enhancing the preservative activity.
\end{abstract}

Keywords-nonionic surfactant; polyol; preservative; ultrafiltration; binding parameter; solubilization; micelle

Many reports have appeared on the inactivation of preservatives in the presence of nonionic surfactants. It is generally accepted that preservative molecules bound within surfactant micelles are inactive, and that the antimicrobial activity is directly related to the concentration of free preservative in the aqueous phase. ${ }^{2)}$ From a practical point of view, some authors $^{3}$ ) reported a potentiating effect of propylene glycol on the antimicrobial activity of preservatives in liquid pharmaceutical preparations. Hibbott ${ }^{4)}$ studied the prevention of spoilage of cosmetic emulsions by microorganisms and found that when propylene glycol was present in the aqueous phase, a more favorable distribution of methyl $p$-hydroxybenzoate between the water and oil phases was attained. Recently, Blanchard ${ }^{5}$ ) reported the effect of sorbitol on the interaction of phenolic preservatives with polysorbate 80 , showing that sorbitol had little effect on the binding of preservatives with surfactant micelles.

The-present paper is eorreermed with the effect of polyols on the interaction between $p$-hydroxybenzoic acid esters and polyoxyethylene (15 units of oxyethylene) dodecyl ether (PDE-15). Glycerol, propylene glycol and 1,3-butylene glycol were studied to determine how these materials function and contribute to the activation of bound methyl $p$-hydroxybenzoate (MP) or propyl p-hydroxybenzoate (PP) within PDE-15 micelles.

\section{Experimental}

Materials-Methyl p-hydroxybenzoate (MP), propyl p-hydroxybenzoate (PP), glycerol and propylene glycol were of J.P. IX grade. Polyoxyethylene (15 units of oxyethylene) dodecyl ether (PDE-15) and 1,3-

1) Location: Juso-Honmachi, Yodogawa-ku, Osaka, 532, Japan.

2) M. Aoki, A. Kamata, I. Yoshioka, and T. Matsuzaki, Yakugaku Zasshi, 76, 939 (1956); N.K. Patel and H.B. Kostenbauder, J.Am. Pharm. Assoc., Sci. Ed., 47, 289 (1958); F.D. Pisano and H.B. Kostenbauder, ibid., 48, 310 (1959); M. Barr and L.F. Tice, ibid., 46, 445 (1957); S.M. Blaug and S.S. Ahsan, J. Pharm. Sci., 50, 138 (1961).

3) M. Barr and L.F. Tice, J. Am. Pharm. Assoc., Sci. Ed., 46, 217 (1957) ; P.S. Prickett, H.L. Murray, and N.H. Mercer, J. Pharm. Sci., 50, 316 (1961).

4) H.W. Hibbott and J. Monks, J. Soc. Cosmetic Chemists, 12, 2 (1961).

5) J. Blanchard, W.T. Fink, and J.P. Duffy, J. Pharm. Sci., 66, 1470 (1977). 
butylene glycol were of commercial grade, supplied by Nihon Emulsion Co., Tokyo and Eastman Organic Chemicals, N.Y., U.S.A., respectively. A Diaflo membrane, UM-10, $43 \mathrm{~mm} \phi$ (cross-linked dextran gel membrane), was purchased from Amicon Corp., Mass., U.S.A.

Measurements of Surfactant Concentration and Critical Micelle Concentration (CMC) - - The concentration of PDE-15 was measured by a surface tension method, and the CMC of PDE-15 was determined by a conventional method, using an ST-1 surface tensometer, Shimadzu Seisakusho, Kyoto.

Ultrafiltration and Quantitative Analysis-C-The binding of p-hydroxybenzoate with PDE-15 was characterized by an ultrafiltration technique and spectrophotometric assay as described previously. ${ }^{6}$ ) The assay procedure of p-hydroxybenzoate presented no problems, since neither PDE-15 nor polyols interfered with the spectrophotometric assay of the preservatives. All procedures were carried out at a temperature of $25^{\circ}$.

\section{Results and Discussion}

\section{CMC of PDE-15}

Plots of the surface tension of PDE-15 solutions against PDE-15 concentration showed a sharp break at a concentration of $6.0 \times 10^{-2} \%$, which corresponded to the CMC. This value is in reasonable agreement with the reported value. ${ }^{\mathbf{a} a, 7)}$

\section{Effect of Polyols on Ultrafiltration}

Aqueous solutions of PDE-15 in the presence and absence of polyols were filtered through a Diaflo UM-10 membrane. Figure 1 shows the surfactant concentration in each fraction. Although a small amount of PDE-15 passed through the membrane, the concentrations in the filtrates were far below the CMC. Thus polyols had little effect on the ability of ultrafiltration to remove PDE-15 micelles from the surfactant solution.

The effect of polyols on the ultrafiltration of $p$-hydroxybenzoate solution was also studied. The plots (Fig. 2) show the $p$-hydroxybenzoate concentration in each fraction from the ultrafiltration in the presence and absence of polyol. As the curves in the presence and absence of polyol coincided well, it appears that polyols had no effect on the ultrafiltration of $p$-hydroxybenzoate solution under these experimental conditions. Thus, ultrafiltration using the Diaflo membrane should be suitable for measurement of the binding characteristics of $p$-hydroxybenzoate-surfactant systems containing polyols.

\section{Effect of Polyols}

There are many ways to express interaction data of preservatives with surfactant micelles. If the preservative concentration bound within the micelles is not proportional to the free preservative concentration in the aqueous phase, simple partition indices have little meaning. ${ }^{6 b, c, 8)}$ However, binding phenomena in under-saturated systems can be represented by the following expression, ${ }^{9}{ }^{\prime}$

$$
r=\frac{\left[D_{\mathrm{b}}\right]}{[S]}=\frac{n \cdot K \cdot\left[D_{\mathrm{f}}\right]}{1+K \cdot\left[D_{\mathrm{f}}\right]}
$$

where $n$ and $K$ are constants corresponding to the number of binding sites and the association constant, respectively; $[S]$ is the concentration of the surfactant; $\left[D_{\mathrm{b}}\right]$ and $\left[D_{\mathrm{f}}\right]$ are the concentrations of bound preservative in the total solution and free preservative in the aqueous phase, respectively; and $r$ is the ratio of bound preservative to surfactant. The value of $\left[D_{\mathrm{b}}\right]$ can be calculated from the data on $\left[D_{\mathrm{f}}\right],[S]$ and the concentration of total preservative

6) a) T. Shimamoto, Y. Ogawa, and N. Ohkura, Chem. Pharm. Bull. (Tokyo), 21, $316(1973)$; b) T. Shimamoto and Y. Ogawa, ibid., 23, $3088(1975)$; c) T. Shimamoto, H. Mima, and M. Nakagaki, ibid., 27, 1995 (1979).

7) P.H. Elworthy and C.B. Macfarlane, J. Pharm. Pharmacol., 17, 65 (1965).

8) M. Donbrow, P. Molyneux, and C.T. Rhodes, J. Chem. Soc. $(A), 1967,561$.

9) E.R. Garrett, J. Pharm. Pharmacol., 18, 589 (1966). 


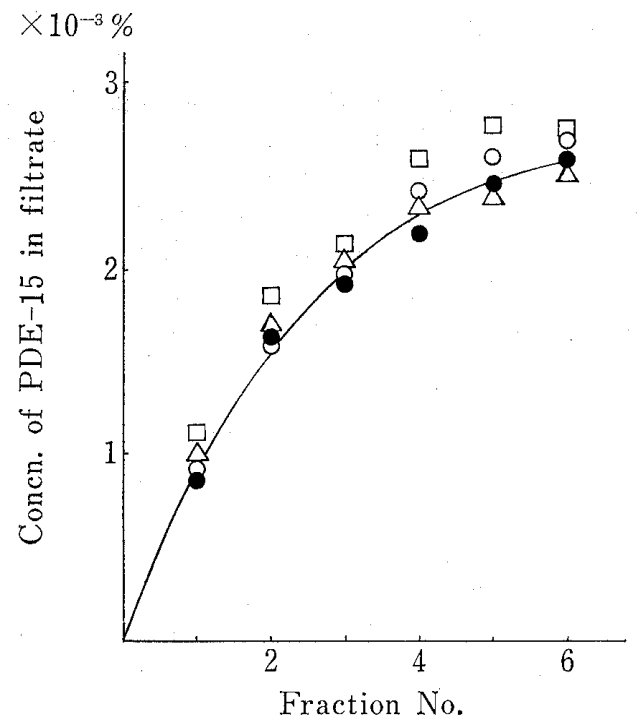

Fig. 1. Ultrafiltration of PDE-15 Solution in the Presence and Absence of Polyols

The filtrates were collected in $4 \mathrm{ml}$ fractions. The PDE-15 concentration of feed solution was $0.5 \%$.

Closed symbols: in the absence of polyol.

Open symbols: in the presence of $1 \%$ polyol

$\bigcirc$, glycerol; $\triangle$, propylene glycol; $\square, 1,3$-butylene glycol.

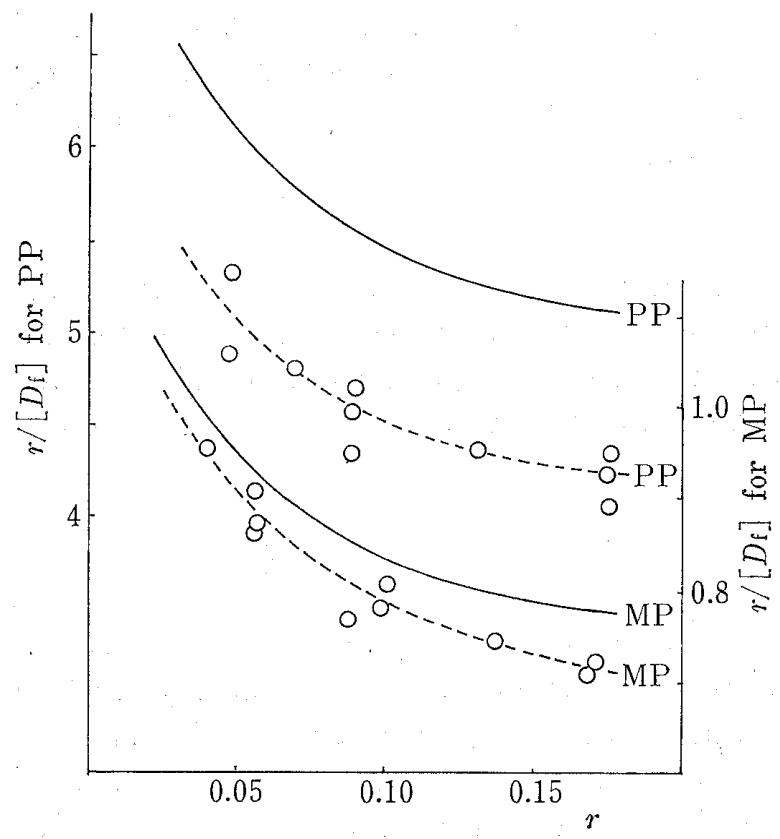

Fig. 3. Scatchard Plots for the Interaction of $p$ Hydroxybenzoate with PDE-15 in the Absence and Presence of Glycerol

The concentrations of PDE-1.5 and glycerol were maintained at $1 \%$.

, in the absence of glycerol;

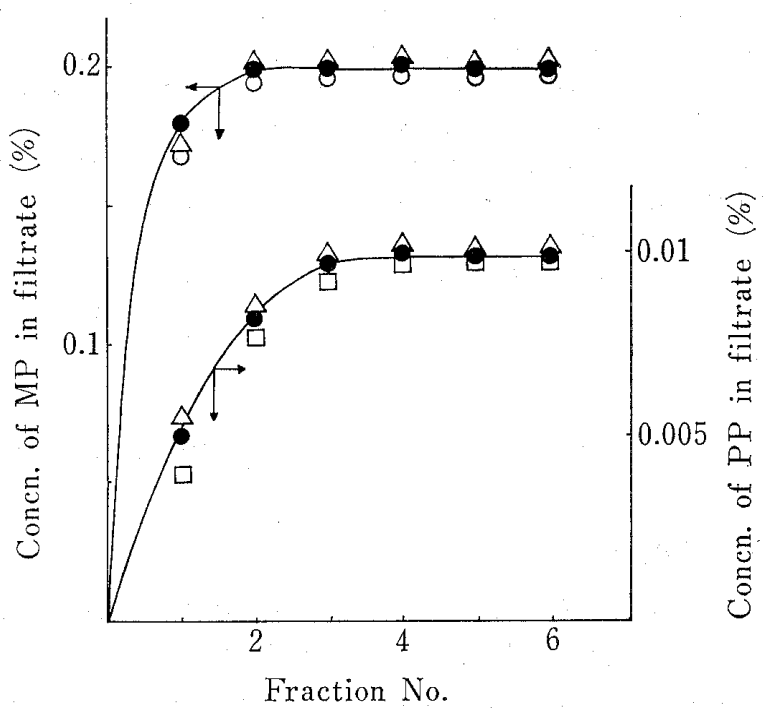

Fig. 2. Ultrafiltration of $p$-Hydroxybenzoate Solution in the Presence and Absence of Polyols

The filtrates were collected in $4 \mathrm{ml}$ fractions. The MP and PP concentrations of feed solutions were $0.2 \%$ and $0.01 \%$, respectively.

Closed symbols: in the absence of polyol.

Open symbols: in the presence of $1 \%$ polyol.

$\bigcirc$, glycerol; $\triangle$, propylene glycol; $\square, 1,3$-butylene glycol.

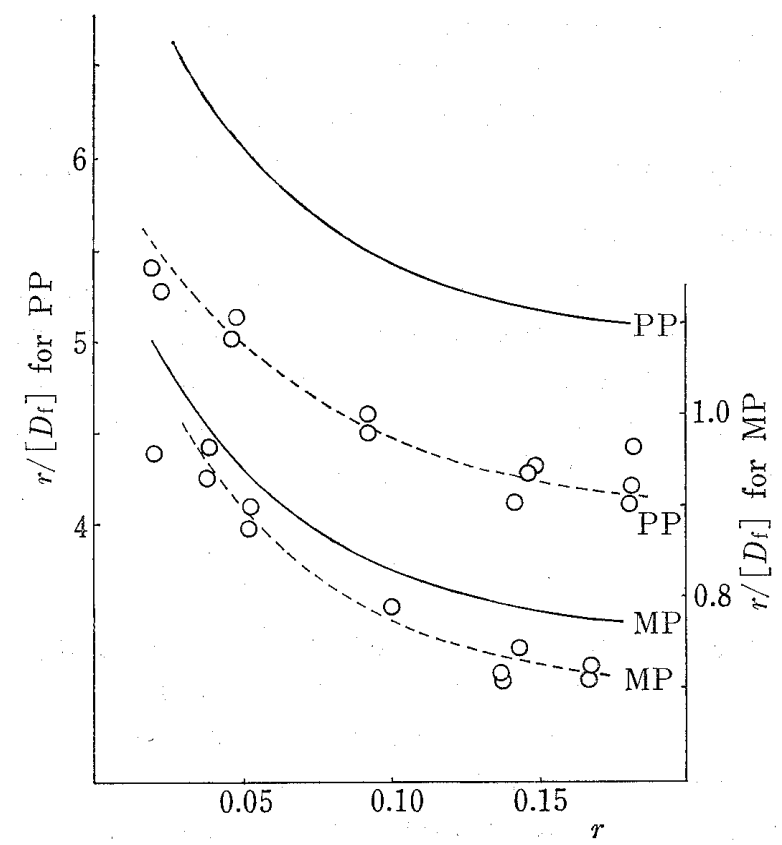

Fig. 4. Scatchard Plots for the Interaction of $p$ Hydroxybenzoate with PDE-15 in the Absence and Presence of Propylene Glycol

The concentrations of $\mathrm{PDE}-15$ and propylene glycol were maintained at $1 \%$.

$\ldots$, in the absence of propylene glycol; 
in the solution, $\left[D_{\mathrm{t}}\right]$, as described previously. $\left.{ }^{6} b\right) \quad$ For graphical treatment of experimental data, a Scatchard plot is generally useful; this can be written as follows,

$$
\frac{r}{\left[D_{\mathrm{f}}\right]}=n \cdot K-r \cdot K
$$

Scatchard plots of the interaction of $\mathrm{MP}$ and PP with PDE-15 in the presence and absence of polyols are shown in Fig. 3,4 and 5 . The concentrations of surfactant and polyol in the sample solutions were both maintained at $1 \%$ so that the ratio of polyol to surfactant was constant in a given experiment. The plots of the binding of $p$-hydroxybenzoate with PDE-15 in the absence of polyol are cited from previous studies. $\left.{ }^{6}, c\right)$ The presence of polyol in the system resulted in a downward displacement of the binding curve, indicating a possible inhibition of the interaction between $p$ hydroxybenzoate and PDE-15 in the presence of polyol.

All the plots were curved, suggesting the presence of more than one class of binding sites. In resolving these complex interactions, data were fitted to a four-parameter model using a modification of the method of Hart ${ }^{10}$ ) and a programmable computer (JEC- 5 spectrum computer). The binding data can be considered in terms of an interaction with two classes of sites, and the calculated parameters are shown in Table I. Thus the interaction in this study was expressed by the following relation.

$$
r=\frac{\left[D_{\mathrm{b}}\right]}{[S]}=\frac{n_{1} \cdot K_{1} \cdot\left[D_{\mathrm{f}}\right]}{1+K_{1} \cdot\left[D_{\mathrm{f}}\right]}+\frac{n_{2} \cdot K_{2} \cdot\left[D_{\mathrm{f}}\right]}{1+K_{2} \cdot\left[D_{\mathrm{f}}\right]}
$$

At high values of $r$, the curve approached a horizontal asymptote, indicating that this class of binding sites has a high capacity but a low affinity, resembling a simple partition process. Since $\left[D_{\mathrm{f}}\right]<0.0145 \mathrm{~mol} / 1$ (for $\mathrm{MP}$ ) or $\left[D_{\mathrm{f}}\right]<0.0019 \mathrm{~mol} / 1$ (for PP) and $K_{\mathrm{2}} \cdot\left[D_{\mathrm{f}}\right] \ll 1$, Eq. (3) could be rewritten as:

$$
r=\frac{\left[D_{\mathrm{b}}\right]}{[S]}=\frac{n_{1} \cdot K_{1} \cdot\left[D_{\mathrm{f}}\right]}{1+K_{1} \cdot\left[D_{\mathrm{f}}\right]}+n_{2} \cdot K_{2} \cdot\left[D_{\mathrm{f}}\right]
$$

The polyols studied here had little effect on the binding of $p$-hydroxybenzoate to the primary class of sites, but in the second class of sites, the values of $n_{2} \cdot K_{2}$ showed a decrease of about $10-15 \%$.

The effects of polyols on the ratios of free to total preservative are shown in Table II. These values were calculated by substituting the binding parameters into Eq. (4). A slight increase in the ratio of free (or available) to total preservative was noted in the presence of polyols, indicating that a more favorable distribution of preservative between water and micelles was obtained. Although no pronounced differences in the effect of polyols could

10) H.E. Hart, Bull. Math. Biophys., 27, 87 (1965). 
TABLE I. Binding Parameters for the Interaction of $p$-Hydroxybenzoate with PDE- $15^{a)}$ at $25^{\circ}$ in the Presence of Polyol ${ }^{a}$ )

\begin{tabular}{|c|c|c|c|c|c|c|c|c|c|c|c|}
\hline \multirow{2}{*}{$\begin{array}{l}p \text {-Hydroxy- } \\
\text { benzoate }\end{array}$} & \multirow{2}{*}{ Polyol } & \multicolumn{2}{|r|}{$n_{1}$} & \multicolumn{2}{|c|}{$K_{1}$} & \multicolumn{2}{|c|}{$n_{2}$} & \multicolumn{2}{|c|}{$K_{2}$} & \multicolumn{2}{|c|}{$n_{2} \cdot K_{2}$} \\
\hline & & $(g / g)$ & $(\mathrm{mol} / \mathrm{mol})$ & $100 \mathrm{ml} / \mathrm{g}$ & $(1 / \mathrm{mol})$ & $(g / g)$ & $\mathrm{col} / \mathrm{mo}$ & $(100 \mathrm{ml} /$ & $(1 / \mathrm{mol})$ & $(100 \mathrm{ml} / \mathrm{s}$ & $(1 / \mathrm{mol})$ \\
\hline \multirow[t]{4}{*}{ MP } & None $^{b)}$ & 0.0223 & 0.124 & 27.0 & 410 & 3.70 & 20.6 & 0.192 & 2.92 & 0.710 & 60.1 \\
\hline & $\mathrm{GL}^{c)}$ & 0.0238 & 0.133 & 27.5 & 418 & 3.30 & 18.4 & 0.200 & 3.04 & 0.660 & 55.9 \\
\hline & $\mathrm{PG}^{c)}$ & 0.0219 & 0.122 & 27.4 & 417 & 3.75 & 20.9 & 0.177 & 2.69 & 0.663 & 56.2 \\
\hline & $\mathrm{BG}^{c)}$ & 0.0222 & 0.124 & 26.6 & 404 & 3.32 & 18.5 & 0.189 & 2.88 & 0.628 & 53.3 \\
\hline \multirow[t]{4}{*}{ PP } & None ${ }^{d)}$ & 0.0230 & 0.108 & 137 & 2480 & 12.3 & 57.6 & 0.373 & 6.71 & 4.58 & 386 \\
\hline & GL & 0.0223 & 0.105 & 126 & 2270 & 10.8 & 50.9 & 0.353 & 6.36 & 3.82 & 324 \\
\hline & PG & 0.0224 & 0.105 & 122 & 2190 & 12.0 & 56.6 & 0.315 & 5.67 & 3.79 & 321 \\
\hline & $\mathrm{BG}$ & 0.0219 & 0.103 & 126 & 2280 & 11.7 & 55.0 & 0.319 & 5.75 & 3.73 & 316 \\
\hline
\end{tabular}

a) The concentrations of surfactant and polyol were maintained at $1 \%$.

b) T. Shimamoto and Y. Ogawa, Chem. Pharm. Bull. (Tokyo), 28,3088 (1975).

c) GL, glycerol; PG, propylene glycol; BG, 1,3-butylene glycol

d) T. Shimamoto, H. Mima and M. Nakagaki, Chem. Pharm. Bull. (Tokyo), 27, 1995 (1979).

TABLE II. Binding of Preservative with PDE- $15^{\alpha)}$ at $25^{\circ}$ in the Absence and Presence of Polyol ${ }^{a}$ )

\begin{tabular}{|c|c|c|c|c|c|}
\hline Preservative & Polyol & $\%$ of $\left[D_{t}\right]^{b)}$ & $\%\left[D_{\mathfrak{f}}\right]$ & $\%$ of $\left[D_{b}\right]$ & {$\left[D_{f}\right] /\left[D_{\mathrm{t}}\right]$} \\
\hline \multirow[t]{16}{*}{$\mathrm{MP}$} & None & 0.350 & 0.195 & 0.157 & 0.557 \\
\hline & & 0.250 & 0.137 & 0.114 & 0.548 \\
\hline & & 0.160 & 0.0851 & 0.0756 & 0.532 \\
\hline & & 0.0800 & 0.0403 & 0.0401 & 0.504 \\
\hline & $\left.\mathrm{GL}^{c}\right)$ & 0.350 & 0.200 & 0.152 & 0.571 \\
\hline & & 0.250 & 0.140 & 0.111 & 0.560 \\
\hline & & 0.160 & 0.0868 & 0.0741 & 0.543 \\
\hline & & 0.0800 & 0.0409 & 0.0396 & 0.511 \\
\hline & $\mathrm{PG}^{c)}$ & 0.350 & 0.201 & 0.151 & 0.574 \\
\hline & & 0.250 & 0.141 & 0.111 & 0.564 \\
\hline & & 0.160 & 0.0874 & 0.0734 & 0.546 \\
\hline & & 0.0800 & 0.0414 & 0.0392 & 0.518 \\
\hline & $\mathrm{BG}^{c)}$ & 0.350 & 0.205 & 0.148 & 0.586 \\
\hline & & 0.250 & 0.144 & 0.108 & 0.576 \\
\hline & & 0.160 & 0.0892 & 0.0716 & 0.558 \\
\hline & & 0.0800 & 0.0422 & 0.0382 & 0.528 \\
\hline \multirow[t]{16}{*}{$\mathrm{PP}$} & None & 0.180 & 0.0291 & 0.151 & 0.162 \\
\hline & & 0.130 & 0.0204 & 0.110 & 0.157 \\
\hline & & 0.0800 & 0.0118 & 0.0683 & 0.148 \\
\hline & & 0.0400 & 0.00543 & 0.0346 & 0.136 \\
\hline & GL & 0.180 & 0.0337 & 0.147 & 0.187 \\
\hline & & 0.130 & 0.0235 & 0.106 & 0.181 \\
\hline & & 0.0800 & 0.0137 & 0.0665 & 0.171 \\
\hline & & 0.0400 & 0.00627 & 0.0338 & 0.157 \\
\hline & $P G$ & 0.180 & 0.0339 & 0.147 & 0.188 \\
\hline & & 0.130 & 0.0237 & 0.106 & 0.182 \\
\hline & & 0.0800 & 0.0138 & 0.0663 & 0.173 \\
\hline & & 0.0400 & 0.00633 & 0.0337 & 0.158 \\
\hline & $\mathrm{BG}$ & 0.180 & 0.0343 & 0.146 & 0.191 \\
\hline & & 0.130 & 0.0240 & 0.106 & 0.185 \\
\hline & & 0.0800 & 0.0140 & 0.0662 & 0.175 \\
\hline & & 0.0400 & 0.00640 & 0.0337 & 0.160 \\
\hline
\end{tabular}

a) The concentrations of PDE-15 and polyol were maintained at $1 \%$.

b) Calculated using the equation, $\left[D_{\mathrm{t}}\right]=\left[D_{\mathrm{b}}\right]+\left[D_{\mathrm{f}}\right](1-[S] / 100)$

c) GI, Glycerol; PG, propylene glycol; BG, 1,3-butylene glycol. 
be found, these results showed that 1,3-butylene glycol was the most effective, while glycerol was somewhat less effective in enhancing the preservative activity. The greatest increase in the ratio was observed at the highest preservative concentration, because polyols influenced only the second class of binding sites.

As has been reported in the previous papers, ${ }^{6}{ }^{6, c)}$ bound preservative molecules are probably located at the junction of the hydrocarbon core and the polyoxyethylene mantle of micelles in the primary class of sites. On the basis of these results, it can be speculated that polyols added to the system are too hydrophilic to penetrate deeply into the micelle and to compete effectively with bound preservative situated at the oxyethylene-hydrocarbon junction. However, polyols modify the binding in the secondary class of sites. It has been assumed that this binding involves a nonspecific partitioning between the micelles and the aqueous phase, and that the preservative molecules are associated with the polyoxyethylene region. ${ }^{6 b, c)}$ The polar polyols are thought to be incorporated into the polyoxyethylene chain part of the micelle structure, displacing a part of the bound preservative from the polyoxyethylene mantle. These considerations are consistent with the relative polarity of the intramicellar regions, polyols and preservative.

Crooks et al. ${ }^{11}$ studied the competitive interaction of preservative mixtures with cetomacrogol, and found that chloroxylenol and dichloroxylenol did not compete significantly with MP in the first class of sites, but that substantial competition occurred in the second class. Blanchard and co-workers ${ }^{5)}$ reported that sorbitol had little effect on the binding of phenolic preservatives with polysorbate 80 in either class of sites when the concentration. of sorbitol added was fairly low compared with the concentration of surfactant. In the present study, the concentrations of polyols used are considered to be sufficient to affect the binding of $p$-hydroxybenzoates in the second class of sites. Based on these results and the foregoing discussion, the interaction with the primary class of sites seems to require considerable structural specificity, while the secondary class of sites appears to interact less specifically. The second class of sites makes the major contribution to the overall binding. Polyols are effective in reducing the inactivation of preservatives by nonionic surfactant by acting at the latter sites, but the effect is not very great.

11) M.J. Crooks and K.F. Brown, J. Pharm. Pharmacol., 26, 235 (1974). 